\title{
Note: In situ cleavage of crystallographic oriented tips for scanning probe microscopy
}

\author{
T. Wutschera) and F. J. Giessibl \\ Institute of Experimental and Applied Physics, University of Regensburg, 93040 Regensburg, Germany
}

(Received 10 November 2010; accepted 10 January 2011; published online 17 February 2011)

\begin{abstract}
We report an in situ method of preparing tips for scanning probe microscopy (SPM). Oriented singlecrystal nickel oxide $(\mathrm{NiO})$ rods were diced, using a wafer saw, to prepare artificial breaking points. Two geometries, a single rod and a two-sided cut rod were fabricated. The cleavable tips were mounted to a force sensor based on a quartz tuning fork and cleaved using the coarse approach of the SPM. Atomically resolved force microscopy images of $\mathrm{NiO}(001)$ were taken with these $\mathrm{NiO}$ tips. (C) 2011 American Institute of Physics. [doi:10.1063/1.3549628]
\end{abstract}

The resolution of scanning probe microscopy (SPM) and the SPM's feedback characteristics depend strongly on the physical nature of the tip and sample. ${ }^{1}$ Recent experiments with well-prepared tips allow the measurement of forces which are necessary to move atoms $\mathrm{s}^{2,3}$ and the imaging of the structure of pentacene with unprecedented spatial resolution. ${ }^{4}$ The atomic configuration and chemical identity of the tip is a key to the distance and angular dependence of tip-sample interactions. ${ }^{5}$

Tip treatment plays a key role in SPM experiments. There are a large number of tip preparation methods such as electrochemical etching, metal coating, annealing, field emission, controlled collision with the sample surface, ion bombardment (see Chap. 13 in Ref. 6), or explosive delamination. ${ }^{7}$ However, these preparation methods require a conductive tip. For nonconductive tip materials, controlled tip collision, sputtering, or metal coating remain as the available preparation tools. Here, the goal was to prepare in situ a clean, crystallographic orientated tip without requiring the previous mentioned preparation methods.

We used mechanical cleavage, a method that works with small crystallites as well as large single crystals to prepare clean tip surfaces. The orientation of preferential cleavage planes is difficult to predict, and several approaches exist that are applicable, depending upon the material. ${ }^{8}$ These include the toughness tensor and the bond density concept. One of the simplest criteria to predict cleavage planes is the bond density concept: silicon and diamond, for example, cleave mainly along $\{111\}$ planes because the number of bonds normal to the surface is minimized here. For cubic structures like $\mathrm{NaCl}$ and $\mathrm{KBr}$, the toughness tensor approach has been shown to be very accurate. The toughness is smallest for the $\{100\}$ planes and the corresponding cleavage planes show a $\{100\}$ orientation. Cleavage can also be affected by defects in the material, either natural or artificial. The artificial defects can be positioned and prepared by diverse material processing techniques like laser treatment, dicing, or milling, depending on the material system.

Nickel oxide was selected as the tip material. It is a cubic system, which crystallizes in rock salt structure and, by

a)Electronic mail: thorsten.wutscher@physik.uni-regensburg.de. observation, cleaves preferentially along $\{100\}$ cleavage planes. Thus three neighboring faces of a cube meet in one corner, that we propose to use as a SPM tip, ideally bounded by one atom with its [111] direction perpendicular to a sample surface. We explored two geometries, a two-sided cut rod and a single rod, as shown in Fig. 1.

The preparation of each geometry started with a lapping process to form small plates of nickel oxide with a thickness between 80 and $450 \mu \mathrm{m}$. A wafer saw (Disco Systems Wafer Saw DAD320) equipped with a thin dicing blade allowed cuts with a minimum width of $35 \mu \mathrm{m} .{ }^{9}$ The nickel oxide plates were attached on an adhesive polymer film during the dicing process. The adhesive strength of the film was chosen according to the geometry, where the simple rod required medium adhesion and the two-sided cut required greater adhesion. ${ }^{10}$

Figure 1(a) shows the simplest geometry (single-cleavetype). A single beam of nickel oxide was cut to dimensions of $0.1 \mathrm{~mm} \times 0.1 \mathrm{~mm} \times 1.7 \mathrm{~mm}$. The length of almost $2 \mathrm{~mm}$ facilitates handling with a tweezer during mounting and enables sufficient torque for cleaving the crystal. The expected fracture pattern of the beam is a simple cleavage perpendicular to the [100] direction. With a clean break, a clean $\{100\}$ surface plane is formed. The point of intersection of the plane and the edge of the rod results in the desired tip.

A two-sided cut nickel oxide rod (dual-cleave-type) is shown schematically in Fig. 1(b). The lateral dimensions are between 300 and $450 \mu \mathrm{m}$. The rod is cut in two neighboring sides. The two splitting planes 1 and 2 prepared by the cuts lead to a predetermined breaking line. In the following, the distance between the end points of the two perpendicular cuts is called a bridge. With the described cutting procedure, minimal bridge widths of $20 \mu \mathrm{m}$ could be obtained. An additional cleavage of the $\{100\}$ plane, plane 3 , resulted in a clean tip out of the volume.

When the geometries were finished they were mounted to a force sensor based on a quartz tuning fork (qPlus sensor), ${ }^{11}$ which provided the base of the in situ tip cleavage. The qPlus sensor can accommodate the large forces acting during cleavage. The qPlus sensor, depicted in Fig. 2, consists of an alumina substrate with electrodes, a quartz tuning fork and the tip. One beam of the quartz tuning fork is glued to the 
(a)

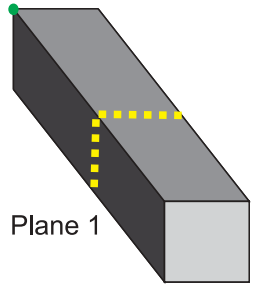

(b)

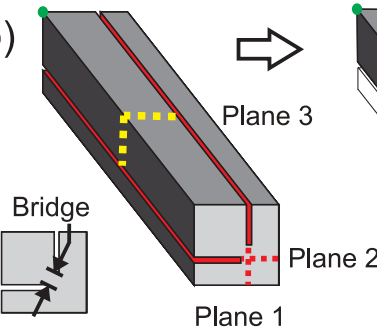

FIG. 1. (Color online) (a) A single nickel oxide rod (single-cleave-type) The dotted line indicates cleavage plane 1 , which arises after cleavage. One corner forms a tip. (b) (Left) The splitting planes of a two-sided diced rod (dual-cleave-type). At first, plane 1 and 2 cleaves. An uncontaminated line is formed which results in a tip with a break along plane 3. (Right) The exposed tip in the former $\mathrm{NiO}$ casing.

substrate. Two types of quartz tuning forks were used, one with a stiffness $k$ of $1820 \mathrm{~N} / \mathrm{m}$ and an eigenfrequency $f_{0}$ of $32 \mathrm{kHz}$ and one with a stiffness $k$ of $4300 \mathrm{~N} / \mathrm{m}$ with an eigenfrequency $f_{0}$ of $60 \mathrm{kHz}$. At the end of the free oscillating quartz beam, the tip was fixed by J-B adhesive weld or Loctite Hysol 1C. ${ }^{12,13}$ With the help of a V-shaped adjustable jig, it was possible to adjust the angle and so the orientation of the prepared crystal rod on the qPlus sensor.

(a)

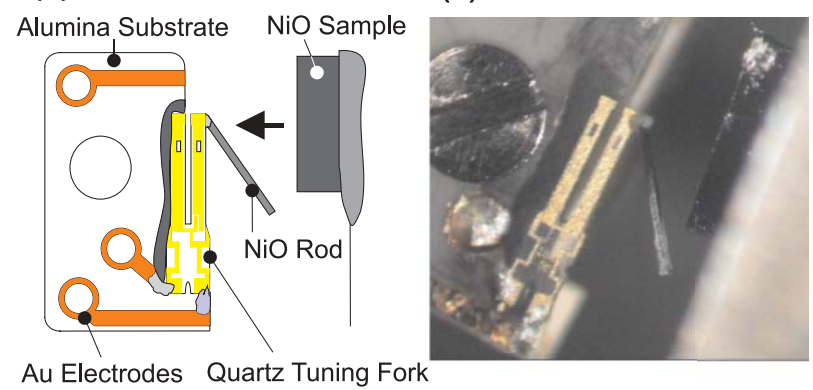

(c)

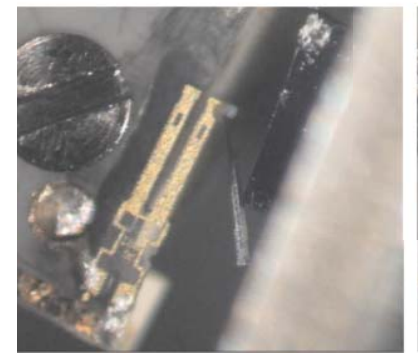

(d)

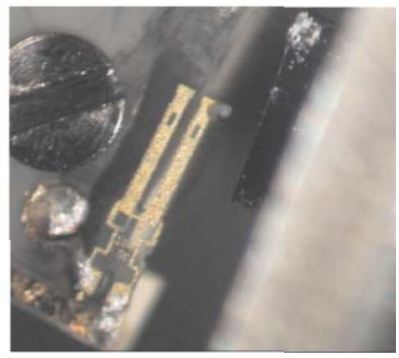

FIG. 2. (Color online) (a) Schematic view of the force sensor with NiO tip rod and sample. On the upper left, the schematic depicts the approach of the sample to the nickel oxide rod, which is glued on the qPlus sensor. (b)-(d) The in situ setup. In (b), the sample approaches to the qPlus sensor until the nickel oxide rod gets in touch with the sample in (c). (d) After a further approach, the tuning fork bends and the nickel oxide rod cleaves.

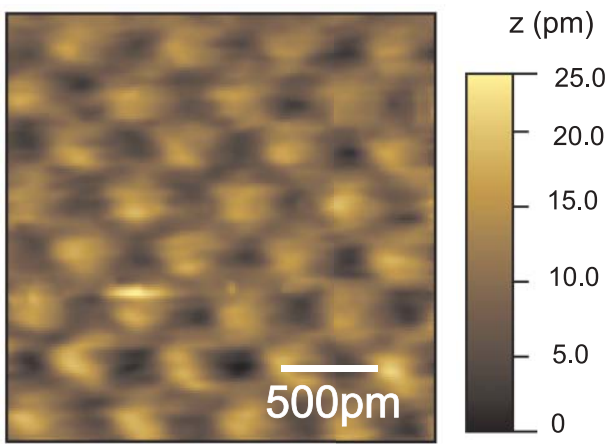

FIG. 3. (Color online) Atomic resolved $2 \mathrm{~nm} \times 2 \mathrm{~nm}$ image of the nickel oxide surface. The topography image was taken at $\Delta f=-7.4 \mathrm{~Hz}$ and an oscillation amplitude $A$ of $1 \mathrm{~nm}$. The qPlus sensor had a resonance frequency of $47326 \mathrm{~Hz}$ and a stiffness of $4300 \mathrm{~m}$.

A modified Autoprobe SP2 microscope was used as a scan head. ${ }^{14}$ The qPlus sensors were held magnetically. The sample sat opposite and was fixed by a spring in a fork on a slip-stick sled for coarse approach. The horizontal design allows for a fast change of samples and tips. The surface was freshly cleaved nickel oxide. ${ }^{15}$ Experiments were performed at room temperature and a pressure of approximately $1.0 \times 10^{-10}$ Torr.

After the positioning of tip and sample the cleavage process was started, as for a single rod (single-cleave-type) shown in Figs. 2(a) -2(b). Figure 2(a) shows a schematic view of the force sensor with NiO tip rod and sample. In Fig. 2(b), the cleavable tip approaches close to the surface. The front of the tip geometry touches with the surface or sample clamps as in Fig. 2(c). A further approach leads to the cleavage [Fig. 2(d)]. Most of the material has broken off, leaving a tip beam with one freshly cleaved surface. The torque that acted during the cleave can be calculated by the stiffness of the tuning fork, the deflection of the prong, and the length of the NiO tip rod. The torque is highest when close to the mounting point. Therefore, the tips are relatively short after cleavage - a desirable result because long tips lead to complications in particular for operation at higher flexural modes. ${ }^{16}$ The deflection during cleavage is measured by recording the cleavage process with a video camera. The maximal possible bending of the free prong corresponds to a force of approximately $240 \mathrm{mN} .^{17}$

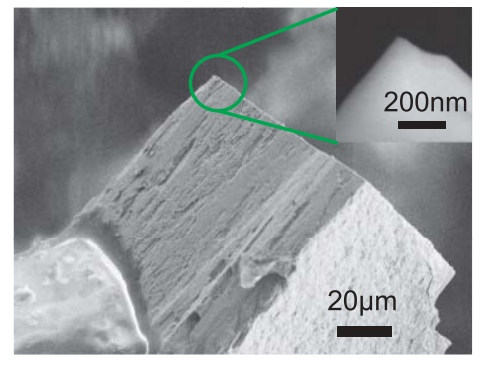

FIG. 4. (Color online) SEM image of a cleaved single rod. The lower side of the rod shows the surface of the lapping process. The left side indicates by longitudinal stripes the diced side of the rod. The magnification shows the tip which has a diameter of $40 \mathrm{~nm}$. 
The forces needed for the cleavage of the single-cleavetype are in general smaller than the maximal force of a bent cantilever. Tuning forks with a stiffness of $4300 \mathrm{~N} / \mathrm{m}$ bent almost unnoticeably during cleavage. Tuning forks with a stiffness of $1820 \mathrm{~N} / \mathrm{m}$ bent approximately $38 \mu \mathrm{m}$ which corresponds to $70 \mathrm{mN}$. The rod length is $1.7 \mathrm{~mm}$, thus the bending moment required for cleavage is $42 \mu \mathrm{Nm}$. In a first approximation, the Euler-Bernoulli beam equation allows the calculation of the stress in the nickel oxide rod. ${ }^{6}$ The stress present in the rod shortly before breaking is around $460 \mathrm{MPa}$. Within nine tip cleavage experiments, the tensile stress at break ranged from 200 to $700 \mathrm{MPa}$, with a median at $335 \mathrm{MPa}$. For comparison, the tensile stress at break for construction steel $\mathrm{S} 235$ is $370 \mathrm{MPa}$.

With the cleaved nickel oxide tip, resulting from a singlecleave-type, atomic resolution was achieved on a nickel oxide sample surface. The interatomic spacing of $\mathrm{NiO}$ (001) is $417 \mathrm{pm} .{ }^{18} \mathrm{~A}$ scanning force microscopy (SFM) topography image is shown in Fig. 3. After the cleavage, the sensor had a resonance frequency of $f_{0} \approx 47326 \mathrm{~Hz}$.

Figure 4 shows a cleaved single rod (single-cleave-type). One side has marks of the dicing blade in the longitudinal direction. The other side has a smoother surface from the lapping process. Ex situ tip radii were measured by Scanning electron microscopy (SEM). From the SEM image (magnification of 383000), a tip radius of $20 \mathrm{~nm}$ was estimated.

With the approach procedure as shown in Fig. 2, the two-sided diced rods [dual-cleave-type, see Fig. 1(b)] were cleaved. Forces applied for these rods were between 110 and $240 \mathrm{mN}$, depending on the width of the bridge. If the bridge was bigger than $30 \mu \mathrm{m}$, cleavage was not possible. An example of the cleavage of double diced rods can be seen in a video linked at Ref. 19.

The prepared tips of the dual-cleave-type were able to resolve the nickel oxide surface, but atomic resolution was not achieved. SEM images showed minimal tip radii of $90 \mathrm{~nm}$.

Cleavage of crystallographic oriented tips in ultra high vacuum (UHV) conditions was shown to produce tips capable of atomic resolution. The method requires highly accurate dicing or milling of the material. Further experiments will focus on the possibility of using focused ion beam (FIB) to produce smaller cleavage structures. This would reduce the forces required to initiate tip cleavage. The in situ tip cleaning process offers uncontaminated tips with known crystallographic orientation for SFM. However, at room temperature, even in a UHV environment, the tips will be contaminated after a few days. Low temperature operation is therefore preferable.

The authors would like to thank Professor T. Peterreins and Dipl. Ing. (FH) F. Steckler for allowing to use the wafer saw. We thank Jochen Mannhart for support. We also thank T. Ascherl and A. Merkel for technical assistance, the colleagues E. Wutscher and A. J. Weymouth for fruitful discussions, and finally the Sonderforschungsbereich 689 for financial support.

${ }^{1}$ S. Hembacher, F. J. Giessibl, and J. Mannhart, Science 53, 380 (2004).

${ }^{2}$ M. Ternes, C. P. Lutz, C. F. Hirjibehedin, F. J. Giessibl, and A. J. Heinrich, Science 319, 1066 (2008).

${ }^{3}$ L. Gross, F. Mohn, N. Moll, G. Meyer, R. Ebel, W. M. Abdel-Mageed, and M. Jaspars, Nat. Chem. 2, 821 (2010).

${ }^{4}$ L. Gross, F. Mohn, N. Moll, P. Liljeroth, and G. Meyer, Science 352, 1110 (2009).

${ }^{5}$ Y. Sugimoto, P. Pou, M. Abe, P. Jelinek, R. Pérez, S. Morita, and Ó. Custance, Nature (London) 446, 64-67 (2007).

${ }^{6}$ C. J. Chen, Introduction to Scanning Tunneling Microscopy (Oxford University Press, Oxford, 1993).

${ }^{7}$ T. Hofmann, J. Welker, and F. J. Giessibl, J. Vac. Sci. Technol. B 28, C4E28 (2010).

${ }^{8}$ R. A. Schultz, M. C. Jensen, and R. C. Bradt, Int. J. Fract. 65, 291 (1994).

${ }^{9}$ NBC-ZH-2050-SE27HDD. NBC-ZH series, Electroformed Bond blades with Hub, Liebigstrasse 8, D-85551 Kirchheim b. München, Germany, www.disco.co.jp.

${ }^{10}$ USI Ultron Systems, Minitron Electronic GmbH. Sägefolie, Type 1003R and $1007 \mathrm{R}$.

${ }^{11}$ F. J. Giessibl, Appl. Phys. Lett. 76, 1470 (2000).

${ }^{12}$ J-B Weld Adhesive, J-B Weld Company, Sulphur Springs, TX.

${ }^{13}$ Loctite Hysol 1C. Ellsworth Adhesives, W129 N10825 Washington Dr., Germantown, WI 53022.

${ }^{14}$ F. Pielmeier, Diploma thesis (Institute of Experimental and Applied Physics, University of Regensburg, 2010).

${ }^{15}$ M. Schmid, A. Renner, F. J. Giessibl, Rev. Sci. Instrum. 77, 036101 (2006).

${ }^{16}$ R. C. Tung, T. Wutscher, D. Martinez-Martin, R. G. Reifenberger, F. J. Giessibl, and A. Raman, J. Appl. Phys. 107, 104508 (2010).

${ }^{17} \mathrm{~T}$. Wutscher, Diploma thesis (Institute of Experimental and Applied Physics, University of Regensburg, 2008).

${ }^{18}$ A. Schwarz, U. D. Schwarz, S. Langkat, H. Hölscher, W. Allers, and R. Wiesendanger, Appl. Surf. Sci. 188, 245 (2002).

${ }^{19}$ See http://www.physik.uni-regensburg.de/forschung/giessibl, 2010. 